\title{
Serum homocysteine and risk of dementia in Japan
}

\section{OPEN ACCESS}

\author{
Sanmei Chen (D) ,' Takanori Honda (D) ,' Tomoyuki Ohara, 1,2 Jun Hata, 1,3,4 \\ Yoichiro Hirakawa, ${ }^{1,4}$ Daigo Yoshida, ${ }^{1}$ Mao Shibata, ${ }^{1,3}$ Satoko Sakata, 1,3,4 Emi Oishi, ${ }^{1,4}$ \\ Yoshihiko Furuta, 1,4 Takanari Kitazono, ${ }^{3,4}$ Toshiharu Ninomiya 1,3
}

\begin{abstract}
- Additional material is published online only. To view, please visit the journal online (http://dx.doi.org/10.1136/ jnnp-2019-322366).
\end{abstract}

${ }^{1}$ Department of Epidemiology and Public Health, Graduate School of Medical Sciences, Kyushu University, Fukuoka, Japan

${ }^{2}$ Department of Neuropsychiatry, Graduate School of Medical Sciences, Kyushu University, Fukuoka, Japan ${ }^{3}$ Center for Cohort Studies, Graduate School of Medical Sciences, Kyushu University, Fukuoka, Japan

${ }^{4}$ Department of Medicine and Clinical Sciences, Graduate School of Medical Sciences, Kyushu University, Fukuoka, Japan

\section{Correspondence to} Dr Toshiharu Ninomiya, Department of Epidemiology and Public Health, Graduate School of Medical Sciences, Kyushu University, 3-1-1 Maidashi, Higashi-ku, Fukuoka 812-8582, Japan; nino@eph. med.kyushu-u.ac.jp

Received 1 November 2019 Revised 6 March 2020

Accepted 23 March 2020 Published Online First 31 March 2020
Check for updates

(C) Author(s) (or their employer(s)) 2020. Re-use permitted under CC BY-NC. No commercial re-use. See rights and permissions. Published by BMJ.

To cite: Chen $\mathrm{S}$, Honda $\mathrm{T}$, Ohara T, et al. J Neurol Neurosurg Psychiatry 2020;91:540-546.

\section{ABSTRACT}

Objective To examine the association between serum total homocysteine levels (tHcy) and dementia risk. Methods A total of 1588 Japanese adults aged $\geq 60$ years without dementia were prospectively followed from 2002 to 2012. Cox proportional hazards models and restricted cubic splines were used to estimate the HRs of tHcy levels on the risk of dementia.

Results During the follow-up, 372 subjects developed all-cause dementia; 247 had Alzheimer's disease (AD) and 98 had vascular dementia (VaD). Compared with the lowest tHcy quintile $(\leq 6.4 \mu \mathrm{mol} / \mathrm{L})$, the multivariableadjusted HRs $(95 \% \mathrm{Cl})$ of the highest quintile $(\geq 11.5$ $\mu \mathrm{mol} / \mathrm{L})$ were 2.28 (1.51-3.43) for all-cause dementia, 1.96 (1.19-3.24) for AD and 2.51 (1.14-5.51) for VaD. In restricted cubic splines, the risk of all-cause dementia steadily increased between approximately 8-15 $\mu \mathrm{mol} / \mathrm{L}$ and plateaued thereafter, with a similar non-linear shape observed for $A D$ and $\mathrm{VaD}$ (all $p$ for non-linearity $\leq 0.02$ ). In stratified analyses by the most recognised genetic polymorphism affecting tHcy concentrations (methylenetetrahydrofolate reductase (677T), the positive association of tHcy with all-cause dementia persisted in both non-carriers and carriers of the risk allele, and even tended to be stronger in the former ( $p$ for heterogeneity=0.07).

Conclusion High serum tHcy levels are associated with an elevated risk of dementia, $\mathrm{AD}$ and $\mathrm{VaD}$ in a non-linear manner, such that an exposure-response association is present only within a relatively high range of tHcy levels. Non-genetic factors affecting serum tHcy concentrations may play important roles in tHcy-dementia associations irrespective of the genetic susceptibility for raised tHcy.

\section{INTRODUCTION}

Raised serum total homocysteine (tHcy) levels have been observed in Alzheimer's disease (AD) ${ }^{1}$ and shown to have direct neurotoxicity ${ }^{2}$ and cause vascular lesions. ${ }^{3}$ These observations have raised questions regarding whether raised serum tHcy is a risk factor for dementia. ${ }^{4}$ This association is of substantial clinical interest because serum tHcy levels are modifiable. ${ }^{56}$ Prospective cohort studies, however, have generated mixed results, with some $e^{7-14}$ showing significant positive associations and others ${ }^{15} 16$ showing none. Moreover, prior studies have merely categorised ${ }^{7-9} 11-15$ or logarithmically transformed ${ }^{79}$ tHcy concentrations to show the magnitude of this association. No studies, to date, have flexibly assessed the specific shape of this association. Indeed, it remains uncertain whether this association is linear or non-linear. Also, prior studies did not fully examine how genetic variation in tHcy metabolism affects the association between serum tHcy levels and risk of dementia.

Therefore, we aimed to address these existing gaps in knowledge by examining the specific shape of the associations between serum tHcy levels and risk of all-cause dementia, $\mathrm{AD}$ and vascular dementia (VaD), and how these associations vary by methylenetetrahydrofolate reductase (MTHFR) C677T polymorphism, a well-recognised genetic polymorphism that has been shown to explain most of the variation in serum tHcy levels attributable to genetic factors. ${ }^{17}$

\section{METHODS}

\section{Study population}

The Hisayama Study, launched in 1961, is an ongoing population-based prospective study of men and women aged 40 years and over residing in the town of Hisayama, a suburb of Fukuoka City in southern Japan. Since the 1960s, the Hisayama population has had age and occupational distributions similar to those of the overall Japanese population. ${ }^{18}$ Health examinations of physical and neurological conditions have been conducted in Hisayama every 1-2 years since 1961. Follow-up surveillance for incident dementia have been performed since 1985, in combination with comprehensive screening surveys for dementia among elderly residents every 6-7 years. ${ }^{19}$

In 2002-2003, a total of 1760 Hisayama residents aged 60 years and over underwent the health examination (participation rate $83.4 \%$ ). Of those, the 1638 attendees who were free of dementia at baseline were included in this study. We excluded 3 participants who did not have the serum tHcy measurement, 45 participants who did not provide information on education and 2 participants who had missing data on serum folate and vitamin $B_{12}$ levels. The final analyses included 1588 participants (676 men and 912 women), whose mean $( \pm S D)$ age was $71 \pm 8$ years (range 60-99). We obtained written informed consent from all participants.

\section{Follow-up survey of dementia}

We followed up the participants for incident dementia from their baseline screening examination to November 2012. As previously reported, ${ }^{19}$ we first established a daily follow-up system comprising the study team, local physicians and members of the town's Health and Welfare Office to collect information on new neurological events, including dementia and stroke. In this surveillance system, physicians of the study team regularly visited hospitals, clinics and 
the town's office to update the information on incident dementia including suspected cases. Second, we repeated neurological and physical examinations at the annual health examination to identify incident dementia cases missed by the surveillance system. For participants who did not participate in the annual health examination or who moved out of the town, we gathered information on their health conditions by postal and telephone surveys. Once dementia or other neurological symptoms were suspected, a psychiatrist and stroke physician from the study team carefully examined the individual for the absence or presence of dementia. Third, to precisely detect dementia cases to the greatest extent possible, we conducted comprehensive neurological and neuropsychological screening for dementia in 2005 and in 2012. When a subject died, we interviewed the family and the attending physician, and reviewed all the available clinical information, including information on cognitive function, neuroimaging (CT/MRI) and activities of daily living. During the follow-up, 346 subjects died and 226 of the decedents underwent brain autopsy; no participants were lost to follow-up.

\section{Diagnosis of dementia}

The diagnosis of each dementia case, including its subtype and the date of diagnosis, was adjudicated by expert stroke physicians and psychiatrists together. The expert stroke physicians and psychiatrists were blind to the subjects' serum tHcy levels. The diagnosis of dementia was made according to the guidelines of the Diagnostic and Statistical Manual of Mental Disorders, 3rd edition, revised. ${ }^{20}$ The diagnoses of $\mathrm{AD}$ and $\mathrm{VaD}$ were made based on the criteria of the National Institute of Neurological and Communicative Disorders and Stroke and the Alzheimer's Disease and Related Disorders Association ${ }^{21}$ and the criteria of the National Institute of Neurological Disorders and Stroke-Association International pour la Recherche et l'Enseignement en Neurosciences, ${ }^{22}$ respectively. Probable or possible or dementia subtypes were defined using the clinical information and morphological examination from neuroimaging. Diagnoses of definite dementia subtypes were made for deceased subjects who underwent autopsy by using neuropathological and clinical information, according to the previously published diagnostic procedure. ${ }^{23}$

\section{Measurements of serum total homocysteine}

Blood samples were centrifuged ( $1500 \mathrm{~g}$ for $5 \mathrm{~min}$ ) after the removal of blood clots at room temperature in half an hour. Separated serum specimens were stored within 3.5-6.0 hours after collection at $-80^{\circ} \mathrm{C}$ until assayed. Serum tHcy levels were analysed in 2005 by using the high-performance liquid chromatography method.

\section{Measurements of covariates}

Information on the following covariates was collected via a questionnaire administered by trained interviewers: educational status, medical history, current treatments of hypertension and diabetes, smoking habits, alcohol drinking habits and regular exercise. History of stroke was determined by using all clinical information prior to the baseline. We measured height and weight in light clothing without shoes and calculated the body mass index (BMI) $\left(\mathrm{kg} / \mathrm{m}^{2}\right)$. Blood pressure was measured three times in a sitting position after a rest of $>5 \mathrm{~min}$. We then calculated the mean systolic and diastolic blood pressure of the three measurements. We defined hypertension as systolic blood pressure $\geq 140 \mathrm{~mm} \mathrm{Hg}$ or diastolic blood pressure $\geq 90 \mathrm{~mm} \mathrm{Hg}$ or current use of antihypertensive agents. We measured plasma glucose levels by using the hexokinase method. We defined diabetes as presenting at least one of the following criteria: fasting glucose level $\geq 7.0 \mathrm{mmol} / \mathrm{L}$, postprandial glucose or 2 hours $75 \mathrm{~g}$ oral glucose postloaded glucose level $\geq 11.1 \mathrm{mmol} / \mathrm{L}$ or use of insulin or oral hypoglycaemic agents. Serum total cholesterol and creatinine levels were measured using the enzymatic method. We calculated the estimated glomerular filtration rate (eGFR) using the Chronic Kidney Disease Epidemiology Collaboration equation with the Japanese coefficient. ${ }^{24}$ Serum albumin levels were measured using the Bromcresol Green method. Serum folate and vitamin $B_{12}$ levels were measured using the chemiluminescence immunoassay method. Genetic analyses, including analysis of the MTHFR C677T polymorphism, were performed by using a HumanOmniExpressExome BeadChip array (Illumina, San Diego, California, USA) at the RIKEN Center for Integrative Medical Sciences. ${ }^{25}$

\section{Statistical analysis}

We tested trends of the baseline characteristics across quintiles of serum tHcy levels by using logistic or linear regression analysis. We calculated the Spearman's correlation coefficients between serum tHcy levels and the MTHFR C677T polymorphism. The incidence rate of dementia were calculated with the person-year method. We used Cox proportional hazards regression models to estimate the HRs and 95\% CIs of dementia according to the quintiles of serum tHcy levels. We first adjusted for age (continuous) and sex (men or women), followed by education ( $\leq 9$ years or $>9$ years), hypertension (yes or no), diabetes (yes or no), serum total cholesterol (continuous), BMI (continuous), eGFR $\left(<60 \mathrm{~mL} / \mathrm{min} / 1.73 \mathrm{~m}^{2}\right.$ or $\left.\geq 60 \mathrm{~mL} / \mathrm{min} / 1.73 \mathrm{~m}^{2}\right)$, history of stroke (yes or no), current smoking (yes or no), current drinking (yes or no), regular exercise ( $<3$ times/week or $\geq 3$ times/week), serum albumin (continuous), serum folate (continuous) and serum vitamin $B_{12}$ levels (continuous, log transformed). The proportional hazards assumption was checked using the log cumulative hazard plots. The trends in dementia risk across the quintiles of serum tHcy were tested by treating the quintiles as a continuous variable and assigning the midpoint concentration for each quintile. We performed sensitivity analyses by excluding individuals who developed dementia within the first 2 years of follow-up $(n=44)$ and those who had not fasted overnight prior to blood sample collection $(n=35)$. We used restricted cubic splines ${ }^{26}$ to show the shape of these associations with four knots placed at the 5th, 35th, 65th and 95th percentiles of serum tHcy levels $(5.0,7.3,9.5$ and $18.2 \mu \mathrm{mol} / \mathrm{L}$, respectively). The fifth percentile $(5.0 \mu \mathrm{mol} / \mathrm{L})$ was set as the reference value. We tested for non-linearity based on the likelihood ratio test by comparing the log-likelihood of the model containing the linear term with that of the model containing cubic spline terms. ${ }^{2627}$

We also ran multivariable-adjusted models stratified by the MTHFR C677T polymorphism to assess the potential effect of this polymorphism on the association between serum tHcy levels and dementia. Thirty participants with missing genotyping data for the MTHFR C677T polymorphism were excluded from this analysis $(\mathrm{n}=1558)$. For the MTHFR C677T polymorphism, subjects with the CT or TT genotype were categorised as carriers, and those with the CC genotype as non-carriers (CC) due to the relatively small proportion of the TT genotype (14\%). We combined the first through the fourth quintiles of serum tHcy into one group (vs the top quintile) to maintain statistical power. The heterogeneity in the associations between subgroups was computed by adding a multiplicative interaction term to the model. We also performed subgroup analyses by all covariates. All statistical analyses were performed using the SAS software (V.9.4; SAS Institute, Cary, North Carolina, USA). Statistical significance was defined as a two-tailed $\mathrm{p}$ value of $<0.05$. 
Table 1 Baseline characteristics of participants according to the quintiles of serum total homocysteine levels

\begin{tabular}{|c|c|c|c|c|c|c|}
\hline \multirow[b]{2}{*}{ Characteristics } & \multicolumn{5}{|c|}{ Serum total homocysteine levels ( $\mu \mathrm{mol} / \mathrm{L}$ ) } & \multirow[b]{2}{*}{$P$ value for trend } \\
\hline & $\begin{array}{l}\text { Quintile } 1 \\
(\leq 6.4) \\
n=329\end{array}$ & $\begin{array}{l}\text { Quintile } 2 \\
(6.5-7.6) n=298\end{array}$ & $\begin{array}{l}\text { Quintile } 3 \\
(7.7-9.0) n=332\end{array}$ & $\begin{array}{l}\text { Quintile } 4 \\
(9.1-11.4) n=312\end{array}$ & $\begin{array}{l}\text { Quintile } 5 \\
(\geq 11.5) \\
\mathrm{n}=317\end{array}$ & \\
\hline Age, years & $68(7)$ & $69(6)$ & $70(7)$ & $72(8)$ & $75(8)$ & $<0.001$ \\
\hline Male, \% & 13.1 & 30.5 & 50.0 & 53.5 & 65.9 & $<0.001$ \\
\hline Education $\leq 9$ years, $\%$ & 45.9 & 49.0 & 53.6 & 55.4 & 59.9 & $<0.001$ \\
\hline Systolic blood pressure, $\mathrm{mm} \mathrm{Hg}$ & $132(20)$ & $137(21)$ & $137(20)$ & $141(22)$ & $140(20)$ & $<0.001$ \\
\hline Diastolic blood pressure, $\mathrm{mm} \mathrm{Hg}$ & $77(10)$ & $80(12)$ & $79(11)$ & $81(12)$ & $80(11)$ & $<0.001$ \\
\hline Use of antihypertensive agents, \% & 25.5 & 35.2 & 33.1 & 41.3 & 45.7 & $<0.001$ \\
\hline Hypertension, \% & 43.8 & 59.4 & 57.2 & 67.6 & 68.8 & $<0.001$ \\
\hline Diabetes, \% & 18.8 & 25.5 & 23.2 & 22.4 & 20.2 & 0.99 \\
\hline Serum total cholesterol, $\mathrm{mmol} / \mathrm{L}$ & $5.5(0.9)$ & $5.4(0.9)$ & $5.2(0.8)$ & $5.1(0.9)$ & $4.9(1.0)$ & $<0.001$ \\
\hline Body mass index, $\mathrm{kg} / \mathrm{m}^{2}$ & $23.1(3.1)$ & $23.5(3.2)$ & $23.1(3.3)$ & $23.0(3.2)$ & $22.4(3.3)$ & $<0.001$ \\
\hline $\mathrm{eGFR}<60 \mathrm{~mL} / \mathrm{min} / 1.73 \mathrm{~m}^{2}, \%$ & 2.7 & 4.4 & 8.1 & 18.3 & 38.2 & $<0.001$ \\
\hline History of stroke, $\%$ & 3.0 & 3.7 & 1.5 & 6.1 & 11.4 & $<0.001$ \\
\hline Current smoking, \% & 7.3 & 7.7 & 14.5 & 21.8 & 21.8 & $<0.001$ \\
\hline Current drinking, $\%$ & 28.3 & 31.9 & 39.5 & 42.6 & 36.3 & 0.002 \\
\hline Regular exercise ( $\geq 3$ times/week), \% & 13.7 & 14.1 & 12.0 & 10.6 & 13.6 & 0.53 \\
\hline Serum albumin, g/dL & $4.3(0.2)$ & $4.3(0.2)$ & $4.3(0.3)$ & $4.3(0.3)$ & $4.2(0.4)$ & $<0.001$ \\
\hline Serum folate, $\mathrm{ng} / \mathrm{mL}$ & $7.1(7.7)$ & $5.8(2.5)$ & $5.2(2.4)$ & $4.9(2.4)$ & $4.3(2.2)$ & $<0.001$ \\
\hline Serum vitamin $B_{12^{\prime}} \mathrm{pg} / \mathrm{mL}^{*}$ & $591(454-871)$ & $502(391-712)$ & 435 (339-572) & 414 (309-575) & $353(247-490)$ & $<0.001$ \\
\hline
\end{tabular}

Data are shown as mean (SD) or frequency.

*Values are median (IQR).

eGFR, estimated glomerular filtration rate;

\section{RESULTS}

The median of serum tHcy levels was $8.3 \mu \mathrm{mol} / \mathrm{L}$ (IQR 6.7-10.6). Table 1 shows baseline characteristics according to the quintiles of serum tHcy concentration. Individuals with a higher serum tHcy level were older, more likely to be men, low-educated, current smokers and current drinkers than those with a lower serum tHcy level. The mean values of systolic and diastolic blood pressure and the rates of use of antihypertensive agents, hypertension, impaired renal function and history of stroke increased significantly across the quintiles of serum tHcy levels. The mean values of serum total cholesterol, BMI, serum albumin, serum folate and serum vitamin $B_{12}$ levels decreased significantly across the quintiles of serum tHcy levels.

During a median follow-up of 10.2 (IQR 7.4-10.3) years and 13591 person-years, 372 participants (135 men and 237 women) developed all-cause dementia. Of those dementia cases, 331 had brain imaging, 72 had brain autopsy and 62 received both procedures; hence, 341 (91.7\%) underwent some kind of morphological examination. Seventeen cases were diagnosed as a mixed type of $\mathrm{AD}$ and $\mathrm{VaD}$, and therefore treated as events in the analysis for each subtype. In all, 247 participants developed $\mathrm{AD}, 98$ developed $\mathrm{VaD}$ and 44 developed other subtypes of dementia. Table 2 shows that the multivariable-adjusted HRs of all-cause dementia, $\mathrm{AD}$ and $\mathrm{VaD}$ were not statistically different for the second through the fourth quintile of serum tHcy levels (HRs ranging from 0.64 to 1.38), but significantly higher (HRs ranging from 1.96 to 2.51 ) for the highest quintile $(\geq 11.5 \mu \mathrm{mol} / \mathrm{L}$; median $14.1 \mu \mathrm{mol} / \mathrm{L})$, as compared with the lowest quintile $(\leq 6.4 \mu \mathrm{mol} / \mathrm{L}$; median $5.7 \mu \mathrm{mol} / \mathrm{L})$. In the sensitivity analyses, the observed associations did not change after we excluded dementia cases occurring within the first 2 years of follow-up (online supplementary table S1) or excluded individuals who did not fast overnight prior to the blood sample collection (online supplementary table S2). In the subgroup analyses by each covariate, there was no evidence of heterogeneity in the association of serum tHcy levels with all-cause dementia (all $\mathrm{p}$ for heterogeneity $\geq 0.10$ ), except with respect to the educational status: the magnitude of the association was significantly stronger in subjects with education $>9$ years than in those with education $\leq 9$ years ( $p$ for heterogeneity $=0.03$ ) (online supplementary table S3).

Figure 1 shows the non-linear trends of these associations from the restricted cubic spline analyses. The risk of all-cause dementia was relatively flat at the low end of the serum tHcy levels, but increased rapidly between around 8 and $15 \mu \mathrm{mol} / \mathrm{L}$ and then plateaued thereafter ( $\mathrm{p}$ for non-linearity $<0.001$ ). A similar non-linear shape was also seen for the tHcy-AD and the tHcy-VaD association ( $p$ for non-linearity $=0.02$ for $A D$ and 0.003 for $\mathrm{VaD}$ ).

The average serum tHcy concentration was higher in carriers (CT/TT) than in non-carriers (CC) of the risk allele of MTHFR C677T (online supplementary table S4, $\mathrm{p}<0.001$ ), with a Spearman's correlation coefficient of $0.1(\mathrm{p}<0.001)$. The MTHFR C677T risk allele was associated with an elevated risk of allcause dementia (online supplementary table S5). Figure 2 shows that the association of serum tHcy with all-cause dementia remained statistically significant in both non-carriers (CC) and carriers (CT/TT) of the risk allele, and tended to be stronger in non-carriers than in carriers ( $p$ for heterogeneity $=0.07$ ). There were no statistically significant differences between non-carriers and carriers in the associations of serum tHcy with $\mathrm{AD}$ and $\mathrm{VaD}$ (both $\mathrm{p}$ for heterogeneity $>0.10$ ).

\section{DISCUSSION}

In this prospective cohort study of Japanese older adults, high serum tHcy levels were associated with an increased risk of allcause dementia, $\mathrm{AD}$ and $\mathrm{VaD}$ in a non-linear manner, such that an exposure-response association was only observed within a range of relatively high serum tHcy levels. The positive association 
Table 2 HRs and $95 \%$ Cls of all-cause dementia and its subtypes according to serum total homocysteine levels

\begin{tabular}{|c|c|c|c|c|c|c|c|}
\hline \multirow[b]{2}{*}{$\begin{array}{l}\text { Serum total homocysteine } \\
\text { levels }(\mu \mathrm{mol} / \mathrm{L})\end{array}$} & \multirow[b]{2}{*}{ No. of events } & \multirow[b]{2}{*}{ Person-years } & \multirow[b]{2}{*}{$\begin{array}{l}\text { Crude incidence rate, } \\
/ 10^{3} \text { person-years }\end{array}$} & \multicolumn{4}{|l|}{ HR $(95 \% \mathrm{Cl})$} \\
\hline & & & & $\begin{array}{l}\text { Age-adjusted and sex- } \\
\text { adjusted }\end{array}$ & $P$ value & Multivariable-adjusted* & $P$ value \\
\hline \multicolumn{8}{|l|}{ All-cause dementia } \\
\hline Quintile $1(\leq 6.4)$ & 54 & 3092 & 17.5 & 1.00 (reference) & & 1.00 (reference) & \\
\hline Quintile 2 (6.5-7.6) & 55 & 2736 & 20.1 & 1.09 (0.75 to 1.59$)$ & 0.64 & 1.13 (0.77 to 1.66$)$ & 0.53 \\
\hline Quintile $3(7.7-9.0)$ & 68 & 3035 & 22.4 & 1.15 (0.80 to 1.65$)$ & 0.46 & 1.25 (0.85 to 1.82$)$ & 0.25 \\
\hline Quintile 4 (9.1-11.4) & 80 & 2620 & 30.5 & 1.31 (0.91 to 1.87 ) & 0.15 & 1.38 (0.93 to 2.03$)$ & 0.11 \\
\hline Quintile 5 ( $\geq 11.5$ ) & 115 & 2109 & 54.5 & 2.14 (1.49 to 3.07 ) & $<0.001$ & 2.28 (1.51 to 3.43$)$ & $<0.001$ \\
\hline$P$ value for trend $t$ & & & & $<0.001$ & & $<0.001$ & \\
\hline \multicolumn{8}{|l|}{ Alzheimer's disease } \\
\hline Quintile $1(\leq 6.4)$ & 40 & 3092 & 12.9 & 1.00 (reference) & & 1.00 (reference) & \\
\hline Quintile 2 (6.5-7.6) & 39 & 2736 & 14.2 & 1.07 (0.68 to 1.66$)$ & 0.77 & 1.13 (0.72 to 1.78$)$ & 0.59 \\
\hline Quintile $3(7.7-9.0)$ & 46 & 3035 & 15.1 & 1.09 (0.71 to 1.69$)$ & 0.68 & 1.12 (0.71 to 1.76$)$ & 0.62 \\
\hline Quintile 4 (9.1-11.4) & 53 & 2620 & 20.2 & 1.20 (0.78 to 1.85$)$ & 0.40 & 1.28 (0.80 to 2.04$)$ & 0.31 \\
\hline Quintile 5 ( $\geq 11.5$ ) & 69 & 2109 & 32.7 & 1.83 (1.18 to 2.82 ) & 0.007 & 1.96 (1.19 to 3.24$)$ & 0.008 \\
\hline$P$ value for trend $t$ & & & & 0.002 & & 0.003 & \\
\hline \multicolumn{8}{|l|}{ Vascular dementia } \\
\hline Quintile $1(\leq 6.4)$ & 14 & 3092 & 4.5 & 1.00 (reference) & & 1.00 (reference) & \\
\hline Quintile 2 (6.5-7.6) & 9 & 2736 & 3.3 & 0.67 (0.29 to 1.56$)$ & 0.35 & 0.64 (0.27 to 1.52$)$ & 0.31 \\
\hline Quintile $3(7.7-9.0)$ & 17 & 3035 & 5.6 & 1.05 (0.51 to 2.17$)$ & 0.89 & 1.37 (0.64 to 2.94$)$ & 0.42 \\
\hline Quintile 4 (9.1-11.4) & 19 & 2620 & 7.2 & 1.17 (0.56 to 2.41$)$ & 0.67 & 1.21 (0.56 to 2.63 ) & 0.63 \\
\hline Quintile 5 ( $\geq 11.5$ ) & 39 & 2109 & 18.5 & 2.67 (1.34 to 5.33$)$ & 0.005 & 2.51 (1.14 to 5.51$)$ & 0.02 \\
\hline$P$ value for trend $t$ & & & & $<0.001$ & & 0.001 & \\
\hline
\end{tabular}

*Adjusted for age, sex, education, hypertension, diabetes, serum total cholesterol, body mass index, estimated glomerular filtration rate, history of stroke, current smoking, current drinking, regular exercise, serum albumin, serum folate and serum vitamin $\mathrm{B}_{12}$ levels (log-transformed).

tTrend tests were conducted by treating the quintiles as a continuous variable and assigning the midpoint concentration for each quintile.

between serum tHcy and all-cause dementia persisted irrespective of the genetic risk for increasing serum tHcy, and even tended to be stronger for those at lower genetic risk for raised serum tHcy. Our findings raise the possibility that a non-linear association exists between the serum tHcy level and dementia.

Our results agree with those from previous prospective studies in which high circulating tHcy levels were associated with an elevated risk of all-cause dementia, ${ }^{7-9}{ }^{11-14} \mathrm{AD}^{7} 810111314$ and VaD. ${ }^{2}$ One meta-analysis of eight cohort studies suggested an exposure-response association between serum tHcy and dementia (HR: 1.5 per $5 \mu \mathrm{mol} / \mathrm{L}$ increment) ${ }^{28}$; however, such a risk estimation depends on the assumption of a linear association. In this study, we first observed that the HRs for all-cause dementia were small and non-significant (1.13 to 1.38$)$ for the second to the fourth quintiles of serum tHcy levels, but more than doubled for the highest quintile. This observed pattern was further confirmed by our analyses of the association shape, which showed that the exposure-response association was not present at the low end of serum tHcy levels, but only within the range of relatively high concentrations (nearly $10 \mu \mathrm{mol} / \mathrm{L}$ and over). The rapid increase in dementia risk in that relatively high serum tHcy range is in line with the findings from several cohort studies ${ }^{71-13}$ and in vitro experiments. ${ }^{2}$ For instance, one study of Japanese older adults found no elevation of dementia risk in the middle (vs the lowest) tertile of tHcy levels, but doubled risk in the highest tertile $(\geq 10.8 \mu \mathrm{mol} / \mathrm{L}){ }^{12}$ In the Framingham Study, the HRs of dementia were not significant in the lower quartiles of tHcy but more than doubled in the highest quartile, despite the use of higher cut-offs of tHcy levels than in the present study. ${ }^{7}$ The MRI cohort study showed that the progression of small vascular lesions was rapidly increased in the highest quintile of serum tHcy levels, but not in the lower quintiles, which could in part explain the non-linear associations we observed in this study. ${ }^{29}$ In previously reported in vitro experiments, the dosedependent neurotoxicity of tHcy occurred at concentrations of $10 \mu \mathrm{mol} / \mathrm{L}$ and over. ${ }^{2}$ Our results, together with these previous reports, suggest that tHcy-lowering interventions (eg, folic acid supplementation, although the evidence is still inconclusive ${ }^{5}$ ) may be effective for reducing the risk of dementia, especially in individuals who have relatively high serum tHcy levels.

We observed a plateau of dementia risk at the high end of serum tHcy levels (around $15 \mu \mathrm{mol} / \mathrm{L}$ and above). It may be that such high serum tHcy levels were sufficiently elevated to exert a maximum effect on dementia risk. However, we cannot rule out the possibility of a monotonic increase in dementia risk after that level of serum tHcy or higher, since an in vitro study showed that serum tHcy at $100 \mu \mathrm{mol} / \mathrm{L}$ seemed to cause more neuronal cell deaths than that at $10 \mu \mathrm{mol} / \mathrm{L}{ }^{2}$ Perhaps, the plateau we observed is attributable to the small number of participants with serum tHcy levels over $15 \mu \mathrm{mol} / \mathrm{L}$ in this study $(\mathrm{n}=127)$. Further largescale prospective studies are warranted to assess the dementia risk at high serum tHcy levels in greater detail.

We found an association between the risk allele of MTHFR C677T and excess risk of dementia, corroborating the results of prior studies. ${ }^{30}$ The MTHFR C677T polymorphism is known to be a common genetic variant that reduces MTHFR activity and consequently increases basal circulating tHcy levels. ${ }^{17} 31$ Raised serum tHcy levels can be caused by both genetic and non-genetic factors (including lifestyle-related factors, diseases and medicines) ${ }^{6}{ }^{17}$ but the former accounts for $<10 \%$ of the variation in circulating tHcy levels. ${ }^{31}{ }^{32}$ In the present study, the difference in the mean value of serum tHcy levels between carriers and non-carriers of the risk allele of MTHFR C677T was modest and the persistent positive associations between serum tHcy and 

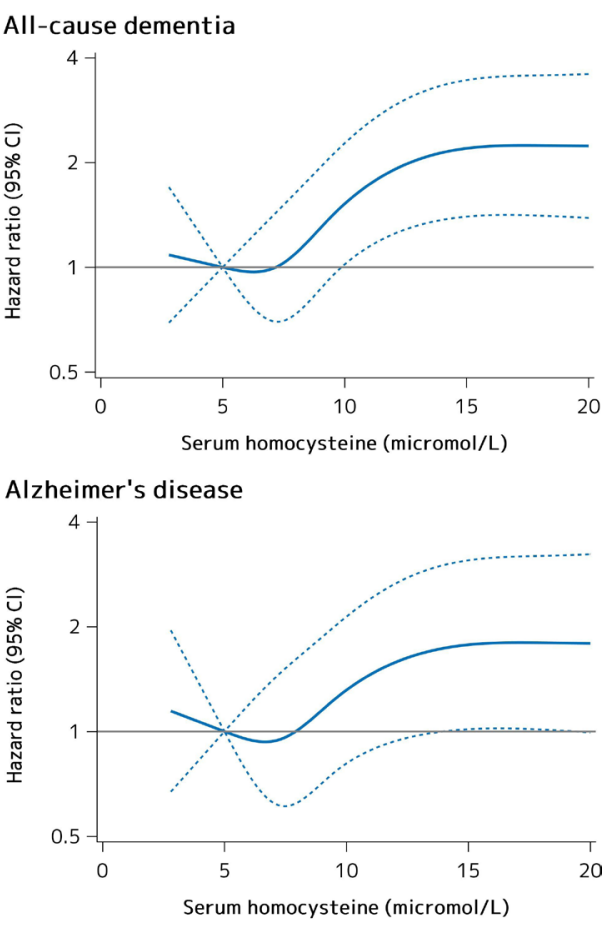

Vascular dementia

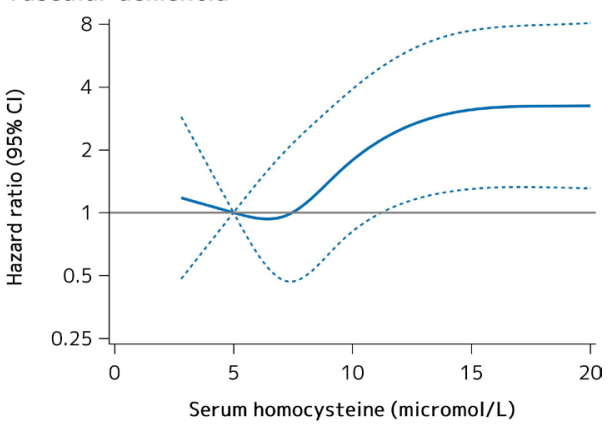

Figure 1 Restricted cubic splines for the association between serum total homocysteine concentrations and risk of dementia and its subtypes. Solid lines represent the HRs; dashed lines represent the 95\% Cls. Knots were placed at the 5th, 35th, 65th and 95th percentiles $(5.0,7.3,9.5$ and $18.2 \mu \mathrm{mol} / \mathrm{L}$ ) of serum total homocysteine. A reference point was set at 5.0 umol/L. Serum total homocysteine values over $20 \mu \mathrm{mol} / \mathrm{L}$ were not present in the plots. The risk estimates were adjusted for age, sex, education, hypertension, diabetes, serum total cholesterol, body mass index, estimated glomerular filtration rate, history of stroke, current smoking, current drinking, regular exercise, serum albumin, serum folate and serum vitamin $B_{12}$ levels (log-transformed).

dementia were observed in both carriers and non-carriers. These findings suggested that non-genetic factors may play important roles in this association irrespective of the genetic susceptibility for raised serum tHcy levels. This leads to the optimistic possibility that individuals at high genetic risk for raised serum tHcy levels might also be targeted in tHcy-lowering interventions to lower their dementia risk.

Raised serum tHcy has long been a well-recognised risk factor for cardiovascular disease. ${ }^{33} 34$ Therefore, the association of tHcy levels with dementia is often explained by vascular mechanisms linked to vascular dementia, ${ }^{32}$ such as impaired endothelial function in the cerebral vasculature ${ }^{335}$ and cerebral amyloid angiopathy. ${ }^{36}$ In addition, homocysteine-induced vascular damages could lead to neuronal death and tau tangle deposition by causing transient ischaemia. ${ }^{37}$ Diet-induced high tHcy levels

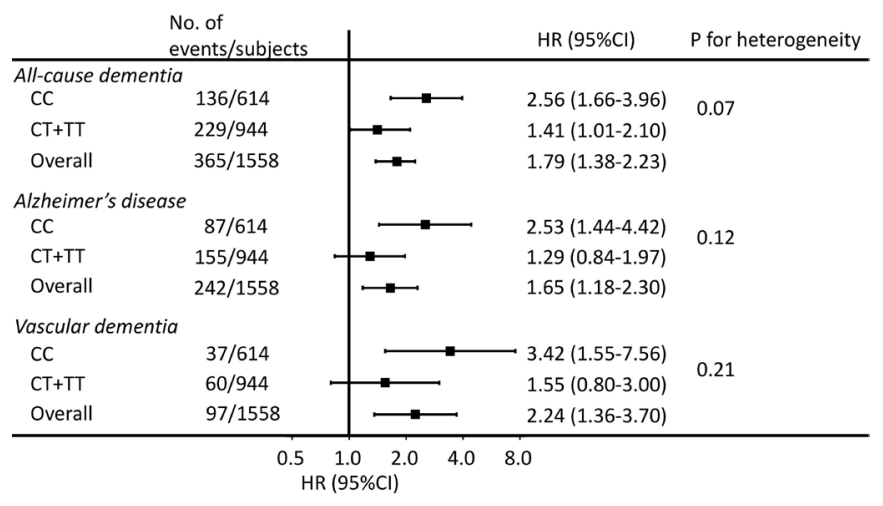

Figure 2 Association between serum total homocysteine levels and dementia and its subtypes stratified by the MTHFR C677T polymorphism. MTHFR, methylenetetrahydrofolate reductase. HRs (95\% Cls) of allcause dementia and its subtypes were estimated for participants in the highest quintile of serum total homocysteine $(\geq 11.5 \mu \mathrm{mol} / \mathrm{L})$ vs those in the lower quintiles $(<11.5 \mu \mathrm{mol} / \mathrm{L})$. The risk estimates were adjusted for age, sex, education, hypertension, diabetes, serum total cholesterol, body mass index, estimated glomerular filtration rate, history of stroke, current smoking, current drinking, regular exercise, serum albumin, serum folate and serum vitamin $B_{12}$ levels (log-transformed).

in the brain have been shown to increase tau hyperphosphorylation, to elevate $\beta$-amyloid production and deposition, and to cause memory deficits in mice. ${ }^{38}$ A population-based autopsy study also showed that elevated circulating tHcy levels were associated with cerebrovascular pathology, and AD pathology. ${ }^{39}$ To sum up, it seems biologically plausible that high serum tHcy levels may increase the risk of all-cause dementia, $\mathrm{AD}$ and $\mathrm{VaD}$ through both vascular and neuronal mechanisms.

We found that the magnitude of the association between serum tHcy and dementia risk was heterogeneous between the education level subgroups, that is, the association was weaker in subjects with a low education level than in those with a high education level. The exact reason for this heterogeneity was unclear. In the present study, for subjects who had a low serum tHcy level, the age-adjusted and sex-adjusted incidence of dementia in those with a low education level was much higher than that in those with a high education level (online supplementary table S6). Therefore, the influence of a low education level, which has been acknowledged to be a strong risk factor for dementia, ${ }^{40}$ may mask the effect on dementia of serum tHcy itself. Nevertheless, the significantly greater dementia risk in subjects with higher serum tHcy levels was observed for both subjects with low and those with high education levels. Thus, this heterogeneity is unlikely to alter our conclusions.

Major strengths of our study include its population-based prospective design in combination with a high participation rate and perfect follow-up, the availability of genetic data and the use of morphological data from neuroimaging and autopsy for accurate diagnosis of dementia subtypes. There are several limitations of this study. First, serum tHcy levels were measured only at baseline, which did not capture the variability during the follow-up. Serum tHcy levels and other covariates may have been changed, such as by folic acid supplement or modifications in lifestyle during follow-up. This may have biassed our results towards the null, resulting in an underestimation of the association between the serum tHcy level and dementia. Second, although we accounted for a wide range of confounders, we cannot rule out residual confounding by unmeasured confounders. Also, residual confounding by metabolic vitamin 
$\mathrm{B}_{12}$ deficiency was still likely to exist after adjustment for serum vitamin $B_{12}$ levels, since the blood concentration of total vitamin $\mathrm{B}_{12}$ does not fully reflect functional deficiency. ${ }^{41}$ Third, there is a possibility that cases of prodromal dementia were more likely to have been included in the subjects with elevated serum tHcy levels at baseline. However, our sensitivity analysis excluding dementia cases occurring within the first 2 years of follow-up did not materially alter any of the results. Fourth, as participants were recruited from one town in Japan, we urge caution in generalising the findings to populations with different backgrounds.

In conclusion, the present study demonstrates that high serum tHcy levels are associated with an elevated risk of dementia, $\mathrm{AD}$ and $\mathrm{VaD}$ in a non-linear fashion, such that an exposure-response association was present only within a relatively high range of serum tHcy levels. In addition, non-genetic factors affecting serum tHcy concentrations may be important in the tHcydementia associations irrespective of the genetic susceptibility for raised serum tHcy levels. Our findings encourage future clinical trials to target individuals who have already presented elevated serum levels of tHcy, in order to test the effect of tHcylowering on cognitive health. In addition, whether such a potential effect on cognitive health would also occur in subjects who are genetically predisposed to high serum tHcy levels warrants further studies.

\section{Twitter Sanmei Chen @sanna_xy and Takanori Honda @pon144}

Acknowledgements The authors would like to thank the residents of the town of Hisayama for their participation in the survey and the staff of the Division of Health and Welfare of Hisayama for their cooperation with this study. The authors would like to thank Professor Yoshinao Oda, Professor Toru Iwaki and the colleagues from the Department of Anatomic Pathology and Department of Neuropathology, Graduate School of Medical Sciences, Kyushu University, who provided very helpful insights and expertise concerning the autopsy findings. The authors conducted statistical analyses by using the computer resources offered under the category of General Projects by Research Institute for Information Technology, Kyushu University.

Contributors SC, TH, TO and TN contributed to the study conception and study design. SC, TH, TO, JH, YH and TN contributed to the data analysis. SC, TH, TO, JH, YH, DY, MS, SS, EO, YF, TK and TN contributed to the data acquisition and interpretation. SC wrote the first draft of the manuscript and all authors contributed to the manuscript revision.

Funding This study was supported in part by Grants-in-Aid for Scientific Research (A) (JP16H02692) and (B) (JP16H05850, JP17H04126 and JP18H02737) and (C) (JP17K09114, JP17K09113, JP17K01853, JP18K07565, JP18K09412 and JP19K07890) and Grants-in-Aid for Early Career Scientists (JP18K17925, JP18K17382 and JP19K19474) from the Ministry of Education, Culture, Sports, Science and Technology of Japan; by Health and Labour Sciences Research Grants of the Ministry of Health, Labour and Welfare of Japan (H29-Junkankitou-Ippan-003 and H30-Shokuhin-[Sitei]-005) and by the Japan Agency for Medical Research and Development (JP19dk0207025, JP19ek0210082, JP19ek0210083, JP19km0405202, JP19ek0210080 and JP19fk0108075).

Disclaimer None of the funding sources had any role in the study design, data analysis, data interpretation, the preparation of the manuscript or the decision to submit the manuscript for publication.

Competing interests None declared.

Patient consent for publication Not required.

Ethics approval This study was approved by the Kyushu University Institutional Review Boards for Clinical Research and for Human Genome/Gene Research.

Provenance and peer review Not commissioned; externally peer reviewed.

Data availability statement Data are available on reasonable request and requires the permission of the Principal Investigator of the Hisayama Study, TN. The datasets used in the present study are not publicly available because confidential clinical data on the study subjects are included.

Open access This is an open access article distributed in accordance with the Creative Commons Attribution Non Commercial (CC BY-NC 4.0) license, which permits others to distribute, remix, adapt, build upon this work non-commercially, and license their derivative works on different terms, provided the original work is properly cited, appropriate credit is given, any changes made indicated, and the use is non-commercial. See: http://creativecommons.org/licenses/by-nc/4.0/.

\section{ORCID iDs}

Sanmei Chen http://orcid.org/0000-0003-0811-1701

Takanori Honda http://orcid.org/0000-0002-1011-9879

\section{REFERENCES}

1 Clarke R, Smith AD, Jobst KA, et al. Folate, vitamin B12, and serum total homocysteine levels in confirmed Alzheimer disease. Arch Neurol 1998;55:1449-55.

2 Lipton SA, Kim WK, Choi YB, et al. Neurotoxicity associated with dual actions of homocysteine at the N-methyl-D-aspartate receptor. Proc Natl Acad Sci U S A 1997;94:5923-8

3 Ikeda U, Ikeda M, Minota S, et al. Homocysteine increases nitric oxide synthesis in cytokine-stimulated vascular smooth muscle cells. Circulation 1999;99:1230-5.

4 Morris MS. Homocysteine and Alzheimer's disease. Lancet Neurol 2003:2:425-8.

5 Homocysteine Lowering Trialists' Collaboration. Lowering blood homocysteine with folic acid based supplements: meta-analysis of randomised trials. BMJ 1998;316:894-8

6 Smith $A D$, Refsum H, Bottiglieri T, et al. Homocysteine and dementia: an international consensus statement. J Alzheimers Dis 2018:62:561-70.

7 Seshadri S, Beiser A, Selhub J, et al. Plasma homocysteine as a risk factor for dementia and Alzheimer's disease. N Engl J Med 2002;346:476-83.

8 Ravaglia G, Forti P, Maioli F, et al. Homocysteine and folate as risk factors for dementia and Alzheimer disease. Am J Clin Nutr 2005:82:636-43.

9 Ford AH, Flicker L, Alfonso H, et al. Plasma homocysteine and MTHFRC677T polymorphism as risk factors for incident dementia. J Neurol Neurosurg Psychiatry 2012;83:70-5

10 Hooshmand B, Solomon A, Kåreholt I, et al. Homocysteine and holotranscobalamin and the risk of Alzheimer disease: a longitudinal study. Neurology 2010;75:1408-14.

11 Kivipelto M, Annerbo S, Hultdin J, et al. Homocysteine and holo-transcobalamin and the risk of dementia and Alzheimers disease: a prospective study. Eur J Neurol 2009;16:808-13.

12 Miwa K, Tanaka M, Okazaki S, et al. Increased total homocysteine levels predict the risk of incident dementia independent of cerebral small-vessel diseases and vascular risk factors. J Alzheimers Dis 2016:49:503-13.

13 Hooshmand B, Refsum H, Smith AD, et al. Association of methionine to homocysteine status with brain magnetic resonance imaging measures and risk of dementia. JAMA Psychiatry 2019. doi:10.1001/jamapsychiatry.2019.1694. [Epub ahead of print: 24 Jul 2019].

14 Zylberstein DE, Lissner L, Björkelund C, et al. Midlife homocysteine and latelife dementia in women. A prospective population study. Neurobio/ Aging 2011;32:380-6.

15 Luchsinger JA, Tang M-X, Shea S, et al. Plasma homocysteine levels and risk of Alzheimer disease. Neurology 2004;62:1972-6.

16 Rastas S, Pirttilä T, Mattila K, et al. Vascular risk factors and dementia in the general population aged $>85$ years. Neurobiol Aging 2010;31:1-7.

17 Bathum L, Petersen I, Christiansen L, et al. Genetic and environmental influences on plasma homocysteine: results from a Danish twin study. Clin Chem 2007:53:971-9.

18 Hata J, Ninomiya T, Hirakawa Y, et al. Secular trends in cardiovascular disease and its risk factors in Japanese: half-century data from the Hisayama study (1961-2009). Circulation 2013;128:1198-205.

19 Ohara T, Hata J, Yoshida D, et al. Trends in dementia prevalence, incidence, and survival rate in a Japanese community. Neurology 2017;88:1925-32.

20 American Psychiatric Association. Diagnostic and statistical manual of mental disorders. 3rd edn, revised. Washington, DC: American Psychiatric Association, 1987

21 McKhann G, Drachman D, Folstein M, et al. Clinical diagnosis of Alzheimer's disease: report of the NINCDS-ADRDA work group under the auspices of department of health and human services Task force on Alzheimer's disease. Neurology 1984;34:939-44.

22 Román GC, Tatemichi TK, Erkinjuntti T, et al. Vascular dementia: diagnostic criteria for research studies. Report of the NINDS-AIREN International workshop. Neurology 1993:43:250-60

23 Fujimi K, Sasaki K, Noda K, et al. Clinicopathological outline of dementia with Lewy bodies applying the revised criteria: the Hisayama study. Brain Pathol 2008; 18:317-25

24 Horio M, Imai E, Yasuda Y, et al. Modification of the CKD epidemiology collaboration (CKD-EPI) equation for Japanese: accuracy and use for population estimates. Am J Kidney Dis 2010:56:32-8

25 Inaishi J, Hirakawa Y, Horikoshi M, et al. Association between genetic risk and development of type 2 diabetes in a general Japanese population: the Hisayama study. J Clin Endocrinol Metab 2019;104:3213-22.

26 Durrleman S, Simon R. Flexible regression models with cubic splines. Stat Med 1989:8:551-61.

27 Govindarajulu US, Spiegelman D, Thurston SW, et al. Comparing smoothing techniques in COX models for exposure-response relationships. Stat Med 2007;26:3735-52. 
28 Wald DS, Kasturiratne A, Simmonds M. Serum homocysteine and dementia: metaanalysis of eight cohort studies including 8669 participants. Alzheimers Dement 2011;7:412-7.

29 Kloppenborg RP, Geerlings MI, Visseren FL, et al. Homocysteine and progression of generalized small-vessel disease: the SMART-MR study. Neurology 2014;82:777-83.

30 Bertram L, McQueen MB, Mullin K, et al. Systematic meta-analyses of Alzheimer disease genetic association studies: the AlzGene database. Nat Genet 2007:39:17-23.

31 Frosst P, Blom HJ, Milos R, et al. A candidate genetic risk factor for vascular disease: a common mutation in methylenetetrahydrofolate reductase. Nat Genet 1995;10:111-3.

32 Smith AD, Refsum H. Homocysteine, B vitamins, and cognitive impairment. Annu Rev Nutr 2016:36:211-39.

33 Homocysteine Studies Collaboration. Homocysteine and risk of ischemic heart disease and stroke: a meta-analysis. JAMA 2002:288:2015-22.

34 Shimizu H, Kiyohara Y, Kato I, et al. Plasma homocyst(e)ine concentrations and the risk of subtypes of cerebral infarction. The Hisayama study. Cerebrovasc Dis 2002;13:9-15.
35 Lai WKC, Kan MY. Homocysteine-induced endothelial dysfunction. Ann Nutr Metab 2015;67:1-12.

36 Li J-G, Praticò D. High levels of homocysteine results in cerebral amyloid angiopathy in mice. J Alzheimer's Dis 2014;43:29-35.

37 Wen Y, Yang S, Liu R, et al. Transient cerebral ischemia induces site-specific hyperphosphorylation of tau protein. Brain Res 2004;1022:30-8.

38 Li J-G, Chu J, Barrero C, et al. Homocysteine exacerbates $\beta$-amyloid pathology, tau pathology, and cognitive deficit in a mouse model of Alzheimer disease with plaques and tangles. Ann Neurol 2014;75:851-63.

39 Hooshmand B, Polvikoski T, Kivipelto M, et al. Plasma homocysteine, Alzheimer and cerebrovascular pathology: a population-based autopsy study. Brain 2013;136:2707-16.

40 Hou X-H, Feng L, Zhang C, et al. Models for predicting risk of dementia: a systematic review. J Neurol Neurosurg Psychiatry 2019;90:373-9.

41 Spence JD. Metabolic vitamin B12 deficiency: a missed opportunity to prevent dementia and stroke. Nutr Res 2016;36:109-16. 